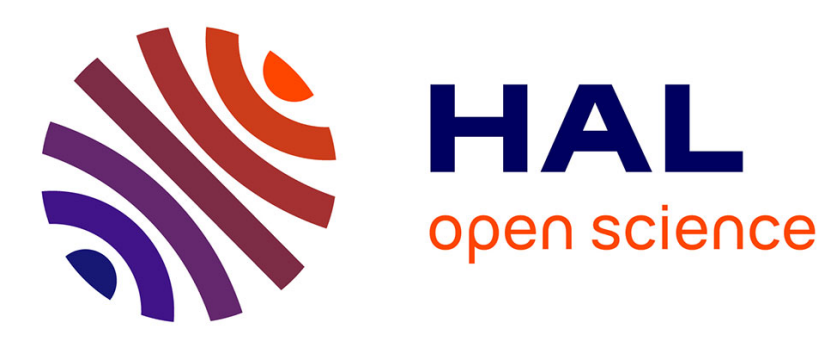

\title{
Nuclear signal simulation applied to gas ionizing chambers
}

\author{
R. Coulon, J. Dumazert
}

\section{To cite this version:}

R. Coulon, J. Dumazert. Nuclear signal simulation applied to gas ionizing chambers. 2015 4th International Conference on Advancements in Nuclear Instrumentation Measurement Methods and their Applications (ANIMMA), Apr 2015, Lisbon, Portugal. pp.7465520, 10.1109/ANIMMA.2015.7465520 . cea-01823372

\section{HAL Id: cea-01823372 https://hal-cea.archives-ouvertes.fr/cea-01823372}

Submitted on 16 Jul 2019

HAL is a multi-disciplinary open access archive for the deposit and dissemination of scientific research documents, whether they are published or not. The documents may come from teaching and research institutions in France or abroad, or from public or private research centers.
L'archive ouverte pluridisciplinaire HAL, est destinée au dépôt et à la diffusion de documents scientifiques de niveau recherche, publiés ou non, émanant des établissements d'enseignement et de recherche français ou étrangers, des laboratoires publics ou privés. 


\title{
Nuclear Signal Simulation Applied To Gas Ionizing Chambers
}

\author{
Romain Coulon and Jonathan Dumazert \\ CEA, LIST, Laboratoire Capteurs et Architectures Electroniques, F-91191 Gif-sur-Yvette, France.
}

\begin{abstract}
Particle transport codes used in detector simulation allow the calculation of the energy deposited by charged particles produced following an interaction. The pulses temporal shaping is more and more used in nuclear measurement into pulse shape analysis techniques. A model is proposed in this paper to simulate the pulse temporal shaping and the associated noise level thanks to the output track file PTRAC provides by Monte-Carlo particle transport codes. The model has been dedicated to ion chambers and more especially for High Pressure Xenon chambers HPXe where the pulse shape analysis can resolve some issues regarding with this technology as the ballistic deficit phenomenon. The model is fully described and an example is presented as a validation of such full detector simulation.
\end{abstract}

Index Terms - Nuclear, Instrumentation, Measurement, Simulation, Monte-Carlo, Ion Chamber, Xenon, Pulse Shape Analysis.

\section{INTRODUCTION}

$\mathrm{N}$ UCLEAR instruments have always to be upgraded in order to address new industrial and societal issues as in research experiment, fuel cycle, electronuclear production, dismantling, environmental, medical and security. Particle transport codes are able to simulate the detector response in term of energy deposition. On another hand, the front-end electronic of such detectors could be designed using electronic simulation tools. In an ideal world, the design of nuclear detector requires to take into account both physics and electronics considerations. However, the results of physical simulations given by Monte-Carlo transport codes cannot directly be used as an input for electronics simulations. Indeed, the pulse signal and its associated noises are shaped by charge productions and migration process and by the coupling to the electronic conversion device. Therefore, a model allowing the pulse temporal shape and its associated noises to be simulated represents an interesting opportunity for future developments in the field of nuclear instrumentations.

Some previews works should be quoted. A pulse shape model has been developed by some laboratories for segmented germanium in order to optimize individual interaction locations using pulse shape analysis [1-3]. Some models have also been developed to simulate the pulse shape of gas detectors as in the case of fission chambers [4] or scintillation in xenon detectors [5]. The model developed in our paper addresses the simulation of ion chambers and more particularly High Pressure Xenon chambers (HPXe).

\section{METHODS}

The current produced by the detector and its front-end electronics can be modeled following the subsequent steps:

- The charge carrier production induced by the interaction between $\gamma$-radiations and the noble gas;

- A current induced at the anode of the detector by the charge carrier motion into the electric field;

- A thermal leakage current and some Gaussian noises added by the components of the charge sensitive preamplifier.

The model is detailed bellow following these three parts.

\section{A. Interaction of $X$ and $\gamma$ rays with matter}

Following the interaction of an X-ray or a $\gamma$-ray into the gas, primary recoil electrons are produced by photoelectric effect, Compton scattering or pair production. Interaction data are calculated using a particle transportation program based on a Monte-Carlo method to solve the Boltzmann transport equations. The MCNPX2.7 code from LANL has been used in this study [6].

A primary recoil electron from interaction is generated into the gas medium. The path of this electron is calculated by the MCNPX program until the cut-off energy of $1 \mathrm{keV}$. The electron loses its energy intermittently due to collisions and continuously due to ionization. Bremsstrahlung radiations can also take place, escaping out of the sensor or creating a secondary recoil electron elsewhere inside the gas. Fig. 1 presents the distribution of the number of collisions calculated over 500 paths of primary electrons with a kinetic energy equal to $662 \mathrm{keV}$ into the xenon gas. The number of collisions is distributed between 40 and 120 collisions with an expected collision number equal to 80 . A density of $0.55{\mathrm{~g} . \mathrm{cm}^{-3}}^{-3}$ and a concentration of $0.2 \%$ of $\mathrm{H}_{2}$ is considered in this study as optimal gas mixture as reported in the literature [7-8]. 


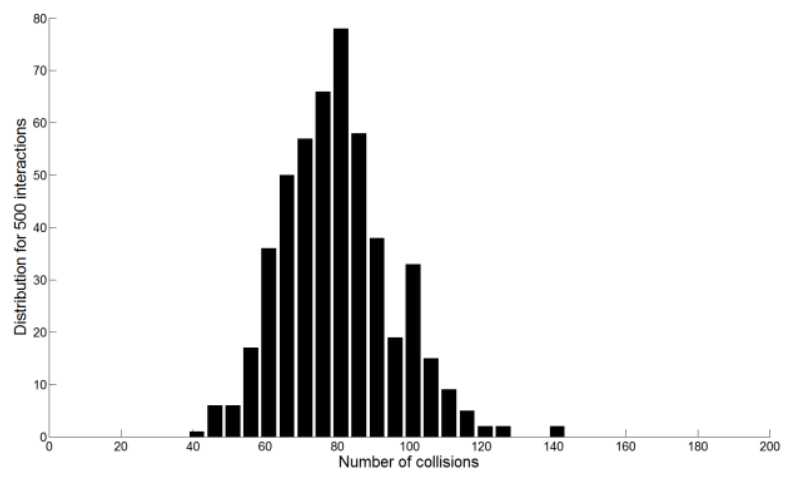

Fig. 1. Distribution of the number of collisions above the energy level of $1 \mathrm{keV}$ calculated over 500 primary electrons with a kinetic energy of $662 \mathrm{keV}$ into the pressurized xenon.

At each collision, a $\delta$-electron is put in motion by recoil, itself slowing down by $\varepsilon$ collisions, ionizations and excitations. The energy threshold for $\delta$ collision lies between $100 \mathrm{eV}$ and 1 $\mathrm{keV}$. $\delta$-electrons have a mean energy around $\mathrm{keV}$ and lose their energy marginally by $\varepsilon$ collisions and mostly by ionizations and excitations. As an illustration, the energy distribution of $\delta$-electrons for $662 \mathrm{keV}$ primary electrons in pressurized xenon is presented on the Fig. 2.

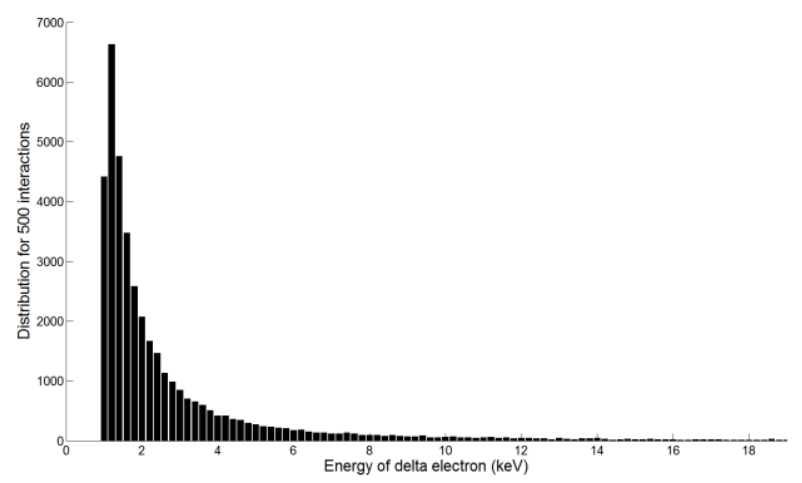

Fig. 2. Distribution of the kinetic energy of $\delta$ electrons into pressurized xenon for a $662 \mathrm{keV}$ primary electron (defined from $1 \mathrm{keV}$ )

The MCNP/PTRAC file containing the details of interaction data associated with primary and secondary electrons is written as an output of the calculation. A post-processing of this file is carried out to model the charge carrier production and migration.

\section{B. Charge carrier production}

The processing of a path history contained into the PTRAC allows the extraction of following data, where $n_{\delta}$ is the number of collisions along the primary recoil electron path:

- $X_{1}^{\delta}=\left(x_{1}^{\delta}, y_{1}^{\delta}, z_{1}^{\delta}\right)$ are coordinates of $\delta$ and $\varepsilon$ collisions

- $X_{2}^{\delta}=\left(x_{2}^{\delta}, y_{2}^{\delta}, z_{2}^{\delta}\right)$ are coordinates of the end of the $\delta$ and $\varepsilon$ electron paths.

- The kinetic energy of the primary electron noted $E_{0}$

- The kinetic energy of $\delta$ and $\varepsilon$ electrons noted $E_{2}^{\delta}$
Fig. 3 shows an example of path of for a $662 \mathrm{keV}$ primary electron into pressurized xenon gas. Red stars represent collision locations and blue lines the path of the primary electron.

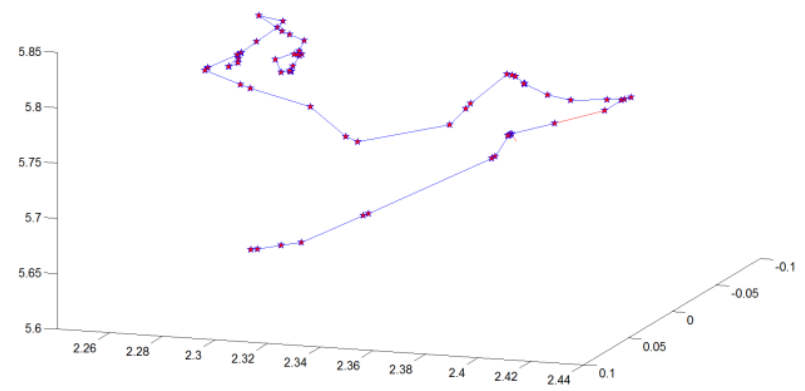

Fig. 3. Path of a $662 \mathrm{keV}$ primary electron in pressurized xenon (unit length in $\mathrm{cm}$ )

At the end of the electron slowing down process, the energy density of electrons is dispatched between the paths of $\delta$ electrons and the path of the primary electron. The dissipated energy by ionization and excitation, $E_{1}^{\delta}$, between every collision is calculated by Eq. 2, where $d_{1}^{\delta}$ is the distance between two successive collisions (see Eq. 1).

$\forall \delta \in\left[1 ; n_{\delta}\right]$

$d_{1}^{\delta}=\left(x_{1}^{\delta+1}-x_{1}^{\delta}\right)^{2}+\left(y_{1}^{\delta+1}-y_{1}^{\delta}\right)^{2}+\left(z_{1}^{\delta+1}-z_{1}^{\delta}\right)^{2}$
$E_{1}^{\delta}=\frac{\left(E_{0}-\sum_{\delta=1}^{n_{\delta}} E_{2}^{\delta}\right) \cdot d_{1}^{\delta}}{\sum_{\delta=1}^{n_{\delta}^{-1}} d_{1}^{\delta}}$

The cooling time of the primary electron is in the order of the picosecond and the ionization occurs in a nanometer range around the track. The energy deposition is therefore considered as punctual in comparison with the duration of charge drifting (in the range of $\mu$ s) studied below. The density of energy is therefore modeled in a linear form following spatial coordinates. A schematic of the 2-D broken line model for recoil electrons is shown in Fig 4. The first dimension $j=1$ describes the path along the primary electron with energy $E_{1}^{\delta}$ uniformly distributed between $X_{1}^{\delta}$ and $X_{1}^{\delta+1}$, the second dimension $j=2$ describes the path along $\delta$ electrons with energies $E_{2}^{\delta}$ uniformly distributed between $X_{1}^{\delta}$ et $X_{2}^{\delta}$.

A schematic of the 2-D broken line model for recoil electrons is shown in Fig 4. 


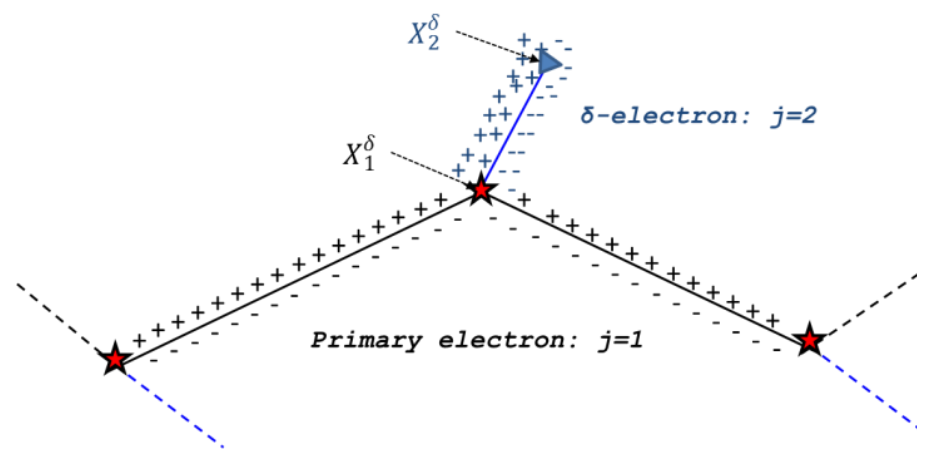

Fig. 4. Schematic of the 2-D broken-line model.

Knowing the energy threshold allowing the product of an electron-ion pair $W_{i}$, expectations associated to the number of charge carrier pair $N_{j}^{\delta}$ created along the primary electron path can be calculated according to Eq. 3:

$\forall \delta \in\left[1 ; n_{\delta}\right], \forall j=\{1 ; 2\}$,

$$
N_{j}^{\delta}=\left\|\frac{E_{j}^{\delta}}{W_{i}}\right\|
$$

The quantification of the number of charges is taken into account using a corrected Poisson sampling described by a Normal distribution $\mathcal{N}$, centered around the expected number of charge $N$ and with an associated variance $F N$ where $F$ is the Fano factor. In our model, random variables $n_{1}^{\delta}$ and $n_{2}^{\delta}$ correspond to the number of charges produced respectively between $\delta$ collisions and along the path of $\delta$ electrons. They are randomly drawn from a Normal distribution $\mathcal{N}$ with expetations $N_{1}^{\delta}$ and $N_{2}^{\delta}$ and variances $F N_{1}^{\delta}$ and $F N_{2}^{\delta}$ as described in Eq. 4.

$\forall \delta \in\left[1 ; n_{\delta}\right], \forall j=\{1 ; 2\}$,

$$
n_{j}^{\delta} \sim \mathcal{N}\left(N_{j}^{\delta}, F N_{j}^{\delta}\right)
$$

As an illustration, Fig. 5 represents the series of expected numbers corresponding to the charge carrier pairs produced along the path of a $662 \mathrm{keV}$ electron interacted with the pressurized xenon.

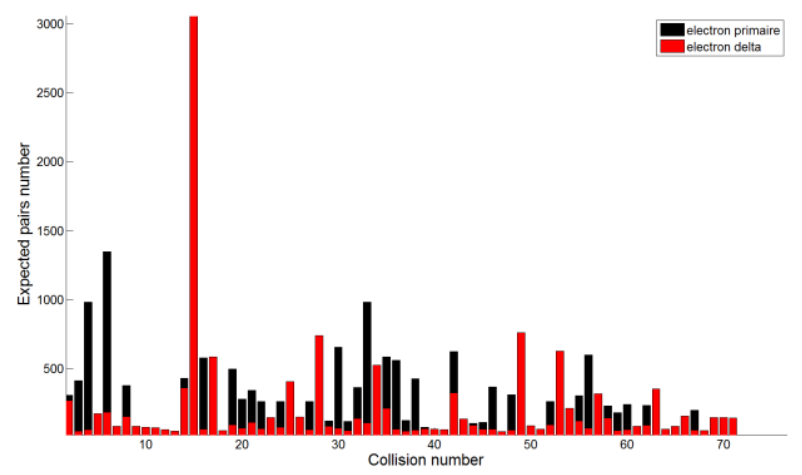

Fig. 5. Expected number of pairs of charge carriers produced for each collision number in the path of a primary electron of $662 \mathrm{keV}$ (back) and for scattered $\delta$ electrons (red).

\section{Charge carriers motion}

Cylindrical coordinates are used to describe the ion chamber as presented in Fig. 6 where $r_{a}$ is the anode radius, $r_{c}$ is the cathode radius, $r_{g}$ is the radius of the Frisch grid and $Z$ the height of the chamber.

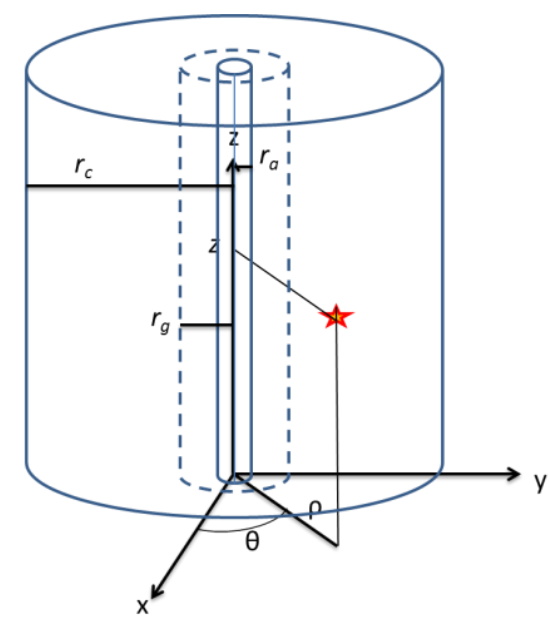

Fig. 6. Geometrical model of the cylindrical ion chamber

The radial positions $\rho_{j, \alpha}^{\delta}$ associated to individual charges $\alpha$ are generated based on a uniform distribution $U$ between two collisions $(j=1)$ or along the delta electron paths $(j=2)$ such as:

$\forall \alpha \in\left[1 ; n_{1}^{\delta}\right], \forall \delta \in\left[1 ; n_{\delta}\right], \forall j=\{1 ; 2\}$,

$\rho_{j, \alpha}^{\delta}=U\left(\sqrt{\left(x_{j, \alpha}^{\delta+1}\right)^{2}+\left(y_{j, \alpha}^{\delta+1}\right)^{2}}, \sqrt{\left(x_{j, \alpha}^{\delta}\right)^{2}+\left(y_{j, \alpha}^{\delta}\right)^{2}}\right)$

A current will be induced by the motion of each individual charge $\alpha$ toward respective electrodes. The collection times $\tau_{\alpha}^{\delta}$ required for charge $\alpha$ to be collected are a random variable generated according to a normal law $\mathcal{N}$ with an expectation $\mathbb{E}\left[\tau_{\alpha}^{\delta}\right]$ corresponding to the time of flight of the considered charge and with a variance $\operatorname{Var}\left[\tau_{\alpha}^{\delta}\right]$ corresponding to a temporal-dispersion term. Indeed, thermal diffusion (Brownian motion) produces a Gaussian dispersion on arrival times whose variance is modeled by the EinsteinSmoluchowski formula. This dispersion is presented in Eq. 620 for electrons $e$ and ions $h$ where $k_{B}$ is the Boltzmann constant and $T$ is the temperature:

$\forall \alpha \in\left[1 ; n_{1}^{\delta}\right], \forall \delta \in\left[1 ; n_{\delta}\right], \forall j=\{1 ; 2\}$ 


$$
\begin{aligned}
& \mathbb{E}\left[\tau_{j, \alpha, e}^{\delta}\right]=\frac{z_{j, \alpha}^{\delta}-z_{c}}{v_{e}} \\
& \mathbb{E}\left[\tau_{j, \alpha, h}^{\delta}\right]=\frac{z_{a}-z_{j, \alpha}^{\delta}}{v_{h}} \\
& \operatorname{Var}\left(\tau_{1, \alpha, e}^{\delta}\right)=\frac{2 k_{B} T\left(z_{1, \alpha}^{\delta}-z_{a}\right)\left(r_{c}-r_{a}\right)}{e V} \\
& \operatorname{Var}\left(\tau_{1, \alpha, h}^{\delta}\right)=\frac{2 k_{B} T\left(z_{c}-z_{1, \alpha}^{\delta}\right)\left(r_{c}-r_{a}\right)}{e V}
\end{aligned}
$$

Charge carriers are susceptible to be trapped by impurities contained into the gas medium. Expected life-times $\theta_{e}$ and $\theta_{h}$ of free charges in the gas are calculated according to Eq. 1011 , where $\Sigma_{e}$ is the cross-section of electron captures, $\Sigma_{h}$ is the cross section of ion captures and $C_{i}$ is the concentration of impurities.

$$
\begin{aligned}
& \theta_{e}=\frac{r_{c}-r_{a}}{C_{i} \Sigma_{e} \mu_{e} V} \\
& \theta_{h}=\frac{r_{c}-r_{a}}{C_{i} \Sigma_{h} \mu_{e} V}
\end{aligned}
$$

The life-time of individual charges is modeled by a random variable generated according to an exponential distribution $\varepsilon$ with parameters $\theta_{e}$ and $\theta_{h}$. Effective collection times $\vartheta_{\alpha}^{\delta}$ are estimated as the minimal value between motion duration and life expectation as seen in Eq. 12-13.

$\forall \alpha \in\left[1 ; n_{1}^{\delta}\right] \& \forall \delta \in\left[1 ; n_{\delta}\right], \forall j=\{1 ; 2\}$,

$$
\begin{aligned}
& \vartheta_{j, \alpha, e}^{\delta}=\min \left\{\mathcal{N}\left(\mathbb{E}\left[\tau_{j, \alpha, e}^{\delta}\right], \operatorname{Var}\left(\tau_{j, \alpha, e}^{\delta}\right)\right) ; \varepsilon\left(\theta_{e}\right)\right\} \\
& \vartheta_{j, \alpha, h}^{\delta}=\min \left\{\mathcal{N}\left(\mathbb{E}\left[\tau_{j, \alpha, h}^{\delta}\right], \operatorname{Var}\left(\tau_{j, \alpha, h}^{\delta}\right)\right) ; \varepsilon\left(\theta_{h}\right)\right\}
\end{aligned}
$$

During their motion, free charges will induce a current on the anode. Shockley-Ramo theorem allows the calculation of this current introducing a weighting field $\xi_{w}$ as described in Eq. 15. This field is obtained from the weighting potential gradient $\varphi_{w}$ presented into the Poisson equation in Eq. 14 as a function of charge density $\boldsymbol{N}$ and the dielectric permittivity $\varepsilon_{0} \varepsilon_{r}$. Equations are solved taking into account, as edge condition, a unit potential applied to the considered electrode (anode) and a null potential to the other electrode $\left(V\left(\rho=r_{c}\right)=0 \& V\left(\rho=r_{a}\right)=1\right)$ :

$$
\begin{array}{ll}
\nabla^{2} \varphi_{w} & =-\frac{\aleph}{\varepsilon_{0} \varepsilon_{r}} \\
\vec{\xi}_{w} & =-\operatorname{grad}\left(\varphi_{w}\right)
\end{array}
$$

Regarding charge motion phenomenon, the problem is invariant by rotation following the $\theta$ angle and by translation following the $z$ axis. The weighting field $\xi_{w}$ is finally calculated in Eq. 16 as a function of the radial position $\rho$ of a charge where $r_{c}$ and $r_{a}$ are the radius of the cathode and the anode:

$$
\xi_{w}=\frac{1}{\ln \left(\frac{r_{c}}{r_{a}}\right) \rho}
$$

Thanks to this weighting field, the currents induced by the electron and ion motion toward the electrodes $i_{e}(t)$ and $i_{h}(t)$ are obtained as functions of time $t \in \mathbb{R}^{+}$such as:

$$
\begin{aligned}
\forall \alpha \in\left[1 ; n_{1}^{\delta}\right], \forall \delta \in\left[1 ; n_{\delta}\right], \forall j & =\{1 ; 2\}, \\
\forall t \in\left[0 ; \vartheta_{j, \alpha, e}^{\delta}\right], \quad i_{j, \alpha, e}^{\delta}(t) & =\frac{e \xi_{w}(t) \mu_{e} V}{r_{c}-r_{a}} \\
& =\frac{e \mu_{e} V}{\ln \left(\frac{r_{c}}{r_{a}}\right)\left[\left(r_{c}-r_{a}\right) \rho_{j, \alpha}^{\delta}-\mu_{e} V t\right]} \\
\forall t \in\left[0 ; \vartheta_{j, \alpha, h}^{\delta}\right], \quad i_{j, \alpha, h}^{\delta}(t) & =\frac{e \xi_{w}(t) \mu_{h} V}{r_{c}-r_{a}} \\
& =\frac{e \mu_{h} V}{\ln \left(\frac{r_{c}}{r_{a}}\right)\left[\left(r_{c}-r_{a}\right) \rho_{j, \alpha}^{\delta}+\mu_{h} V t\right]}
\end{aligned}
$$

$\forall t \in\left[\vartheta_{j, \alpha, e}^{\delta} ;+\infty\right], \quad i_{j, \alpha, e}^{\delta}(t)=0$

$\forall t \in\left[\vartheta_{j, \alpha, e}^{\delta} ;+\infty\right], \quad i_{j, \alpha, h}^{\delta}(t)=0$

In the presence of a Frisch grid, the current is solely induced on the anode when the electrons pass through the grid. The time for electron to achieve the grid $\tau_{g}$ and electronic and ionic currents $i_{j, \alpha, e}^{\delta}(t), i_{j, \alpha, h}^{\delta}(t)$ are presented in Eq. 21-23:

$\tau_{g}=\frac{\left(\rho_{j, \alpha}^{\delta}-r_{g}\right)\left(r_{c}-r_{a}\right)}{\mu_{e} V}$

$\forall \tau_{g}>0$,

$\forall t \in\left[0 ; \tau_{g}\right], \quad i_{j, \alpha, e}^{\delta}(t)=0$

$\forall t \in[0 ;+\infty], \quad i_{j, \alpha, h}^{\delta}(t)=0$

When the electron reaches the grid, it can be trapped by the grid with a probability $p_{g}$ (typically $1 \%$ ). A Bernoulli variable $X$ is set equal to 1 with a probability $p_{g}$ and 0 with a probability $\left(1-p_{g}\right)$. Finally, the signal resulting from charge collection is determined by summing the contribution of every elementary charge generated after each collision summed overall collisions. The electronic current, the ionic current and the total current are calculated as shown in Eq. 25-27:

$$
\begin{aligned}
i_{e}(t) & =\sum_{j=1}^{2} \sum_{\delta=1}^{n_{\delta}} \sum_{\alpha=1}^{n_{1}^{\delta}} i_{j, \alpha, e}^{\delta}(t) \\
i_{h}(t) & =\sum_{j=1}^{2} \sum_{\delta=1}^{n_{\delta}} \sum_{\alpha=1}^{n_{1}^{\delta}} i_{j, \alpha, e}^{\delta}(t) \\
i(t) & =i_{e}(t)+i_{h}(t)
\end{aligned}
$$




\section{Electronics}

The detector is coupled to a charge sensitive preamplifier allowing the charge integration and delivering an output voltage which can be processed by analog or digital electronics (see Fig. 7).

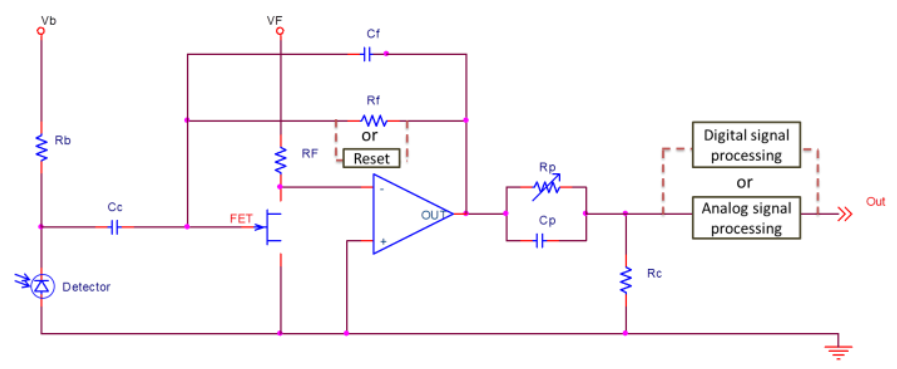

Fig. 7. Schematic of front-end electronics

Charge integration is performed by the preamplifier whose time constant $\tau_{f}$ has to be high enough compared to the electron collection time $\tau_{e}$. Eq. 28-29 show the calculation of $\tau_{f}$ and $\tau_{e}$, where $R_{f}$ and $C_{f}$ are the feedback resistance and capacitance.

$$
\begin{aligned}
\tau_{e} & =\underset{t}{\arg \min }\left[i_{e}(t)\right] \\
\tau_{f} & =R_{f} C_{f}
\end{aligned}
$$

The equivalent signal charge $Q_{S}$ is a function of the charge transfer efficiency $\eta$, which depends on the ratio between the detector capacitance $C_{d}$ and the FET gate source capacitance $C_{g s}$. Eq. 30 details the calculation of the detector capacitance as a function of the absolute and relative dielectric constant $\varepsilon_{0}$ and $\varepsilon_{r}$ in the cylindrical detector:

$C_{d}=\frac{2 \pi \varepsilon_{0} \varepsilon_{r} Z}{\ln \left(\frac{r_{c}}{r_{a}}\right)}$

The calculation of the charge transfer efficiency $\eta$ is detailed in Eq. 31:

$$
\eta=\frac{1}{1+\frac{C_{d}}{C_{g s}}}
$$

The current from the detector is integrated by the preamplifier and then differentiated to obtain a differential output current $i_{d}(t)$ as shown in Eq. 34. The integration and differentiation impulse responses $h_{i}(t)$ and $h_{d}(t)$ are presented in the Eq. 32-33, where $\tau_{d}$ is the time constant of the differentiator circuit $\left(\tau_{d} \ll \tau_{f}\right)$.

$$
\begin{aligned}
h_{i}(t) & =\frac{1}{\tau_{f}} \exp \left(-t / \tau_{f}\right) \\
h_{d}(t) & =\delta(t)-\frac{1}{\tau_{d}} \exp \left(-t / \tau_{d}\right) \\
i_{d}(t) & =\eta\left(\left(i * h_{i}\right) * h_{d}\right)(t)
\end{aligned}
$$

The signal can be processed by an analog or digital circuit. The first strategy regarding the triggering optimization and SNR maximization is the implementation of a linear shaping filter with a triangular impulse response $h_{s}(t)$ presented in Eq. 35. This filter is close to the optimal cups filter and it is then largely implemented into analog and digital electronics $[9,10]$. It is defined by the zeros-to-peak time constant $\tau_{m}$ such as:

$$
h_{s}(t) \begin{cases}=1-\frac{|t|}{t_{m}} & \forall t \in\left[-t_{m} ; t_{m}\right] \\ =0 & \forall t \in\left[-\infty ;-t_{m}\left[\cup\left[t_{m} ;+\infty\right]\right.\right.\end{cases}
$$

The equivalent output current $i_{s}(t)$ obtained by this filtering is described in Eq. 36.

$$
i_{s}(t)=\left(i_{d} * h_{s}\right)(t)
$$

Finally, the equivalent source charge $\hat{Q}_{s}$ is estimated by the maximum of the equivalent output current $i_{s}(t)$ which is an estimation of the deposited energy $E_{0}$ where $k$ is a calibration factor.

$\hat{E}_{0}=k \hat{Q}_{s}=k \max \left(i_{s}(t)\right)$

On the other hand, Digital Signal Processing unit (DSP) allows the implementation of nonlinear filtering approaches. These methods are able to maximize the SNR as efficiently as linear filters without any pulse shaping $[11,12]$. The equivalent output current $i_{d}(t)$ is filtered into an anti-aliasing analog circuit before its digitalization thanks to an Analog to Digital Convertor (ADC). The estimated charge $\hat{Q}_{s}^{\prime}$ is therefore obtained by a simple integration of equivalent current pulse $i_{d}(t)$ as presented in Eq. 38 .

$\hat{E}_{0}=k \hat{Q}_{s}^{\prime}=k \int_{t=0}^{+\infty} i_{d}(t) d t$

Thanks to the high bandgap of noble gases, the leakage current induced by thermal production of free-charges can be considered as null $\left(\exp \left(-E_{g} / k_{B} T\right)=2 \cdot 10^{-174}\right)$. The noise arising from the insulator surface and the noise coming from electromagnetic compatibility (EMC) are considered as environmental perturbations and are not deal with in this study. The digital noise (quantification and sampling) induced by the DSP is also considered as null as it is not a challenge with currently available ADC [13]. It is then considered that the major part of the electronic noise comes from electronic components located at the first stage of the preamplifier. Indeed, the electronic noise can be divided in two parts: a parallel noise and a serial noises:

Parallel noise is the noise induced by components set in parallel with regards to the preamplifier entrance. It is itself composed by Johnson-Nyquist noises thermally induced into the bias and the feedback resistances ( $R_{b}$ and $R_{f}$ respectively); 
and a shot noise induced by the bias current $V_{b} / R_{b}$. The equivalent noise charge coming from parallel noises $E N C_{p}$ is calculated as presented in the Eq. 39:

$E N C_{p}^{2}=\left[\frac{1.34 k_{B} T\left(R_{f}+R_{b}\right)}{R_{f} R_{b}}+\frac{2 e V_{b}}{R_{b}}\right] \tau_{m}$

The contribution of this noise decreases when the bandwidth increases. It has to be maintained as low as possible by setting the parallel resistors at very high values. This requirement follows the same direction as the necessity to ensure full charge integration (ensured by $\tau_{f} \gg \tau_{e}$ ), but roses the risk of preamplifier saturation when the pulse rate increases. The electron collection time is especially high in large gas chambers, which imposes to implement high value for the feedback resistance. The use of transistor reset preamplifiers is a more efficient way to operate with a quasi-infinite feedback resistance and managing of the saturation phenomenon. The parallel noise could then be considered as low compared to the serial noise contribution $E N C_{p}^{2} \ll E N C_{s}^{2}$.

The serial noise arises from a thermal leakage current into the FET itself and a flicker noise (ie. 1/f noise). The equivalent noise charge from serial noise $E N C_{s}$ is calculated as presented in Eq. 40 where $g_{m}$ is the transconductance and $K_{f}$ the flicker noise constant of the FET component:

$E N C_{s}^{2}=\left(C_{d}+C_{g s}\right)^{2}\left[\frac{8 k_{B} T}{3 g_{m} \tau_{m}}+\frac{K_{f}}{C_{g s}}\right]$

The FET has to be chosen with a high transconductance $g_{m}(5 \mathrm{mS})$ and low flicker energy constant $K_{f}\left(10^{-27} \mathrm{~J}\right)$. A compromise has to be made with regards to the gate-source capacitance. Indeed, high values increase the charge transfer efficiency $\eta$, but also the serial noise contribution. Matching by $C_{d} \approx C_{g s}$ allows an optimization to be made. The thermal part of the serial noise increases with the bandwidth, whereas the flicker part is independent from it. In linear filters, the time constant $t_{m}$ has to be set large enough to decrease the thermal noise under the flicker noise level. However, the system dead time will increase dramatically with the shaping time constant increasing. Nonlinear filtering implementable into DSP then constitute a better strategy to deal with SNR maximization without any pulse shaping.

The noise charge $Q_{n}$ is calculated using the total equivalent noise charge as a standard deviation of a normal law as shown in Eq. 41.

$Q_{n} \sim \mathcal{N}\left(0, \sqrt{E N C_{p}^{2}+E N C_{s}^{2}}\right)$
This full detector simulation allows to maximize the signal to noise ratio with regards to both physical and electronics parameters.

Interaction of a $662 \mathrm{keV}$ photon into an HPXe chamber is simulated by MCNPX and the processing of the output PTRAC file is subsequently run. An example of the current induced by the charge carriers at the anode is presented in Fig 6. We can observe that the Frisch grid reduces the dependence on the interaction location.

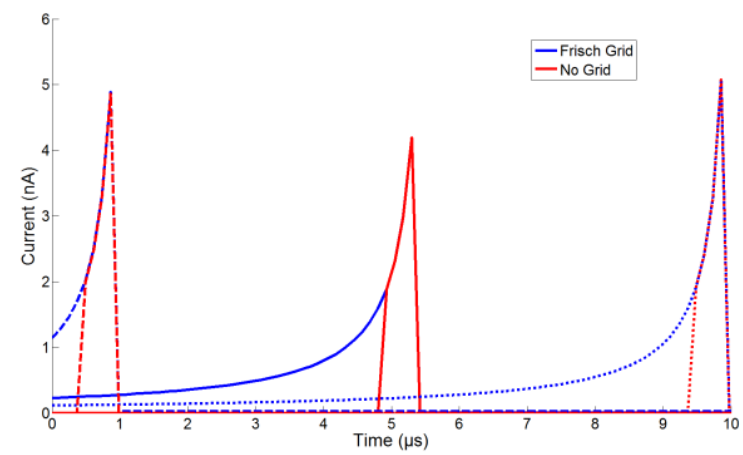

Fig. 8. Example of pulse shape $i_{d}(t)$ measured at the output of the preamplifier, close to the anode (long dash line), between anode and cathode (full line) and close to the cathode (small dash line).

The signal measured at the output of the shaper is shown in Fig. 9.

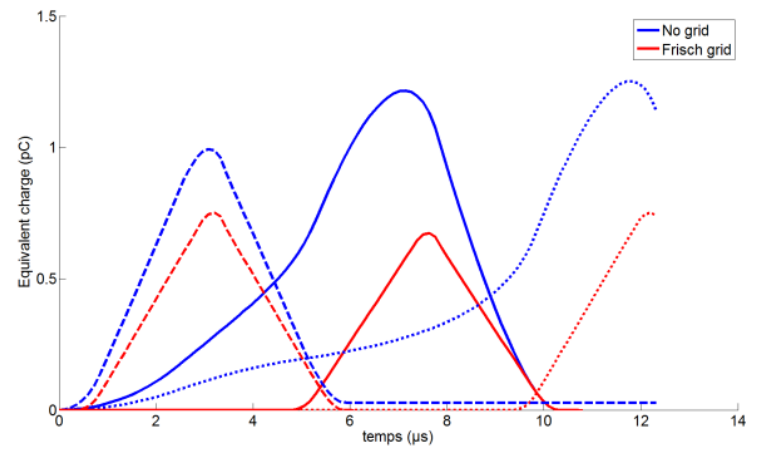

Fig. 9. Example of pulse shape $i_{s}(t)$ measured at the output of the shaper, close to the anode (long dash line), between anode and cathode (full line) and close to the cathode (small dash line).

A gamma spectrum has been measured with an HPXe chamber filled with a xenon density of $0.25 \mathrm{~g}_{\mathrm{ccm}}{ }^{-3}\left(0.3 \% \mathrm{H}_{2}\right.$, $113 \mathrm{~mm}$ diameter and $170 \mathrm{~mm}$ length). The measure has been simulated by MCNPX code in order to produce the related PTRAC file. The measured spectrum and the deposited energy spectrum (tally f8) obtained by simulation are shown in Fig. 10. Simulated and experimental spectra show a relatively good accordance proving that the physics of the interaction is well processed in MCNPX code. The lack of counts at low energy could be explained by the fact that all scattered photons are not taken into account in the current version of our model. A Gaussian broadening card (GEB card) has been implemented in order to match the simulation with the experiment adding an artificial resolution. We should notice here that the parameter $\mathrm{a} b \mathrm{c}$ of the Gaussian broadening (see Eq. 42) could only be set a posteriori with regards to the experimental observation. 


$$
F W H M(E)=a+\sqrt{b E+c E^{2}}
$$

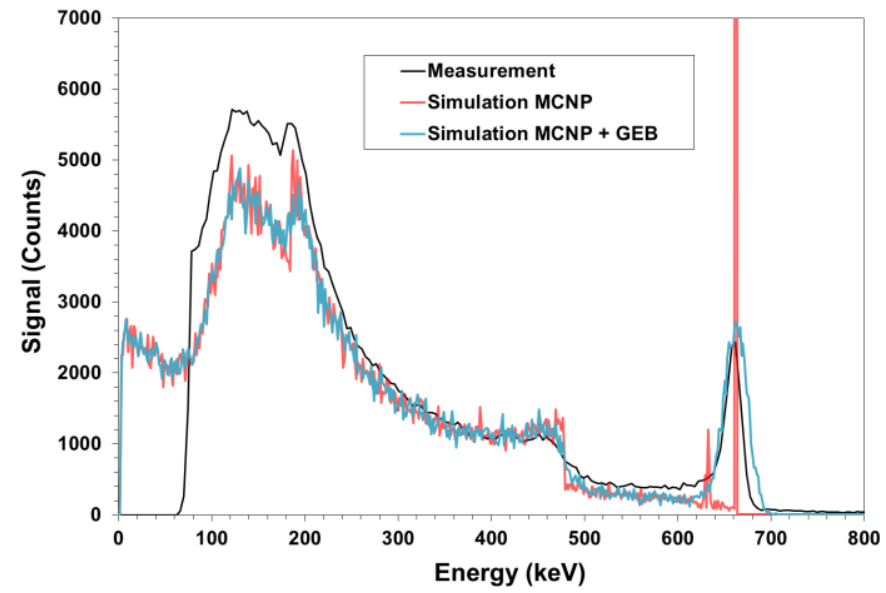

Fig. 10. Gamma spectrum obtained for an HPXe chamber with a xenon density of $0.25{\mathrm{~g} . \mathrm{cm}^{-3}}^{-3}\left(0.3 \% \mathrm{H}_{2}, 113 \mathrm{~mm}\right.$ diameter and $170 \mathrm{~mm}$ length $)$.

Fig. 11 shows the pulse high spectrum obtained thanks to the computation of the PTRAC file by the pulse model simulation presented above. The blue spectrum is the spectral response with the intrinsic resolution of the detector where only the statistical fluctuation of charge carrier number is taken into account). The green spectrum is the spectral response with intrinsic resolution and dispersions due to charge motion effects as charge recombination, grid trapping, thermal diffusion and ion motion toward to cathode. The Frisch grid allows a robust limitation of the resolution discrepancy thanks to the shielding of the ionic part of the signal. The electronic noise is finally added and the red spectrum represents the signal as it can be measured by such a detector. It can be seen that this final spectrum is close to the experimentally obtained one. This result constitutes a first validation of the full detector simulation.

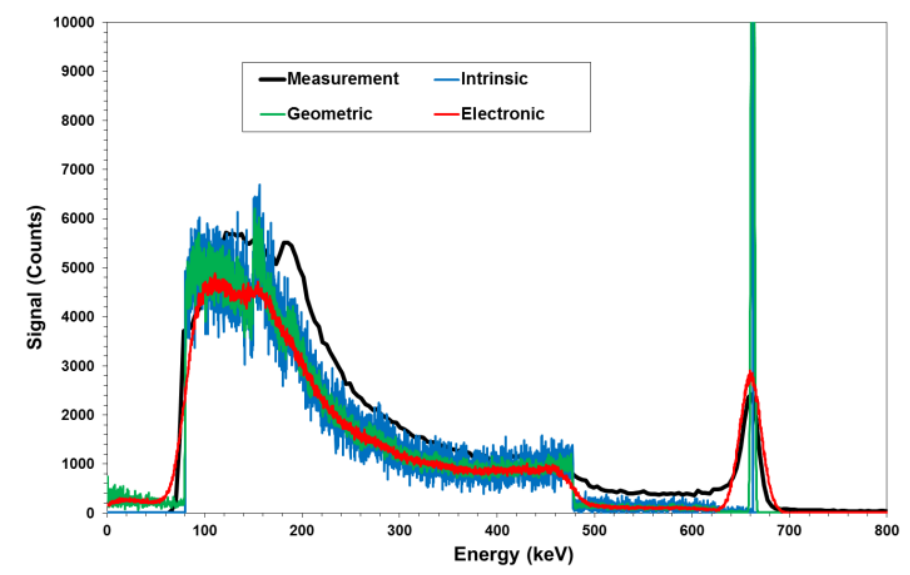

Fig. 11. Gamma spectrum obtained for an HPXe chamber with a xenon density of $0.25{\mathrm{~g} . \mathrm{cm}^{-3}}^{-3}\left(0.3 \% \mathrm{H}_{2}, 113 \mathrm{~mm}\right.$ diameter and $170 \mathrm{~mm}$ length $)$.

\section{DISCUSSION}

As individual pulses can be simulated, it is now possible to simulate the temporal series of interaction events. The general model is presented in Eq. 43 for a series of pulses $k$ where $i_{s}^{k}(t)$ is the temporal shape of individual pulse and $\tau_{k}$ the inter-pulses period. The period $\tau_{k}$ is calculated in Eq. 44 by sampling an exponential distribution $\mathcal{E}$ with an expected interpulse period $T$.

$$
\begin{aligned}
& j_{s}(t)=\sum_{k>1} i_{s}^{k}\left(t-\tau_{k}\right) \\
& \tau_{k} \sim \mathcal{E}(T)
\end{aligned}
$$

Traditionally the temporal signal is simulated without taking into account the individual shape of the stochastic pulse series. The pulse is decomposed in charge amplitude $Q_{k}$ and a general model for the pulse impulse response $H(t)$ as seen in Eq. 45.

$$
i_{s}^{k}(t)=Q_{k} H(t)
$$

Therefore, the signal could be simulated in a better way in Eq. 43 in order to study pile-up or fluctuation phenomena [14]. This implementation will be explored in future works.

\section{CONCLUSION}

A model allowing the simulation of the overall ion chamber detector has been described. MCNPX simulation has been completed by the processing of the output PTRAC file. At each individual interaction, the production and the migration of the charge carriers has been simulated to format every individual pulse shape. The associated electronic noise has also been taken into account according to electronic parameters of the detector and front-end electronics.

The simulation has been compared with experimental data obtained by an HPXe chamber prototype. The good agreement between experimental and simulation constitute a first validation of this simulation technique applied to radiation detectors. This approach gives the opportunity to optimize both physical and electronics parameters for $\mathrm{R} \& \mathrm{D}$ in the field of nuclear instrumentation.

\section{REFERENCES}

[1] M. Kurokawa, S. Shimoura, H. Iwasaki, H. Baba, S. Michimasa, S. Ota, H. Murakami and H. Sakai, "Pulse Shape Simulation and Analysis of Segmented Ge Detectors for Position Extraction." IEEE Transactions on Nuclear Science, 50(5): 1309-1316, 2003.

[2] I. Abt, A. Cadwell, D. Lenz, J. Liu, X. Liu, and B. Majorovits, "Pulse shape simulation for segmented true- 
coaxial HPGe detectors." The European Physical Journal C 68: 609-618, 2010.

[3] M. Schlarb, R. Gernhäuser, S. Klupp, and R. Krücken, "Pulse shape analysis for $\gamma$-ray tracking: Pulse shape simulation with JASS." The European Physical Journal A 47: 132, 2011.

[4] P. Filliatre, C. Jammes, B. Geslot, and R. Veenhof, "A Monte Carlo simulation of the fission chambers neutroninduced pulse shape using the GARFIELD suite." Nuclear Instruments and Methods in Physics Research A 678: 139147, 2012.

[5] J. Mock, N. Barry, K. Kazkaz, D. Stolp, M. Szydagis, M. Tripathi, S. Uvarov, M. Woods, N. Walsh. "Modeling Pulse Characteristics in Xenon with NEST." Journal of Instrumentation 9, T04002, 2014.

[6] G.W. McKinney, et al. "MCNPX 2.7.0 New Features Demontrated" - Los Alamos National Laboratory technical report LA-UR-12-25775, 2012.

[7] V.V. Dmitrenko, A.S. Romanyuk, S.I. Suchkov, and Z.M. Uteshev. Electron mobility in dense xenon gas. Soviet Physics Technical Physics, 28:1440-1444, 1983.

[8] A. Bolotnikov and B. Ramsey. The spectroscopic properties of high-pressure xenon. Nuclear Instruments and Methods in Physics Research A, 396:360-370, 1997.

[9] V. Radeka. “ Low-noise techniques in detectors." Annual Reviews of Nuclear and Particle Science 38: 217-277, 1988.

[10] V.T. Jordanov, G.F. Knoll, A.C. Huber and J.A. Pantazis. "Digital techniques for real-time pulse shaping in radiation measurements ". Nuclear Instruments and Methods in Physics Research A 353 261-264, 1994.

[11] E. Barat, T. Dautremer, T. Montagu and J-C. Trama. "A bimodal Kalman smoother for nuclear spectrometry". Nuclear Instruments and Methods in Physics Research A 353 261-264, 1994.

[12] Y. Moline, M. Thevenin, G. Corre and M. Paindavoine. "Auto-Adaptive Trigger and Pulse Extraction for Digital Processing in Nuclear Instrumentation". IEEE Transaction on Nuclear Science, submitted 2015.

[13] S. Normand, et. al. "PING for Nuclear Measurements: First Results", IEEE Transactions on Nuclear Science, 59(4): 1232-1236, 2012.

[14] T. Rebafka, F. Roueff and A. Souloumiac. "Information bounds and MCMC parameter estimation for the pile-up model". Journal of Statistical Planning and Inference, 141: 116, 2011. 